McGill Law Journal

Revue de droit de McGill

\title{
LE DROIT EST-IL UNE ARME?
}

\section{Laurence Bich-Carrière}

Volume 62, numéro 2, december 2016

URI : https://id.erudit.org/iderudit/1040055ar

DOI : https://doi.org/10.7202/1040055ar

Aller au sommaire du numéro

Éditeur(s)

McGill Law Journal / Revue de droit de McGill

ISSN

0024-9041 (imprimé)

1920-6356 (numérique)

Découvrir la revue

Citer ce document

Bich-Carrière, L. (2016). LE DROIT EST-IL UNE ARME?. McGill Law Journal/

Revue de droit de McGill, 62(2), 567-574. https://doi.org/10.7202/1040055ar d'utilisation que vous pouvez consulter en ligne.

https://apropos.erudit.org/fr/usagers/politique-dutilisation/ 


\section{LE DROIT EST-IL UNE ARME?}

\section{Laurence Bich-Carrière*}

\section{LA VERSION PRONONCÉE FAIT FOI}

Mesdames, messieurs les juges, public chéri, mon amour,

Le droit est-il une arme?

Il est vaste le magasin de la métaphore et le bel esprit pouvait y puiser encore que le droit est une rapière parce qu'il permet de faire mouche de finesse, une arme nucléaire, selon l'expression consacrée en matière d'injonction Mareva1 ${ }^{1}$, un lance-pierre, aussi, pensant à David et Goliath,

* BCL/LLB McGill 2008, LLM Cantab 2009, Barreau du Québec 2009, Barreau du Haut Canada 2011; avocate, Lavery de Billy, SENCRL. Allocution prononcée le 3 décembre 2015 à Cotonou, au Bénin, dans le cadre du concours de plaidoirie de la trentième Conférence internationale des barreaux de tradition juridique commune. Le discours, sur la position imposée d'une réponse négative à la question "Le droit est-il une arme? », a mérité la première place à son auteure.

(c) Laurence Bich-Carrière 2016

Citation: (2016) 62:2 McGill LJ 567 — Référence : (2016) 62:2 RD McGill 567

1 Voir Bank Mellat v Nikpour (1982), [1985] FSR 87 à la p 92, [1982] Com LR 158 (CA), juge Donaldson :

The rule requiring full disclosure seems to me to be one of the most fundamental importance, particularly in the context of the draconian remedy of the Mareva injunction. It is in effect, together with the Anton Piller order, one of the law's two "nuclear" weapons. If access to such a weapon is obtained without the fullest and frankest disclosure, I have no doubt at all that it should be revoked.

L'injonction Mareva, qui permet de veiller à ce que les actifs d'un défendeur ne s'évanouissent pas en fumée (et rappelons ici que le Mareva est un cigare que l'on appelle parfois aussi "petit corona" au moment de mettre un jugement à exécution). Elle tire son nom de l'affaire Mareva Compania Naviera SA v International Bulkcarriers SA, [1975] 2 Lloyd's Rep 509, [1980] 1 All ER 213 (CA), Lord Denning. On l'appelle depuis, au Royaume-Uni, le freezing order. Au Canada, elle apparaît d'abord en Ontario dans l'affaire Chitel v Rothbart (1982), 141 DLR (3e) 268, 39 OR (2e) 513 (CA Ont), puis est reconnue par la Cour suprême dans Aetna Financial Services Ltd c Feigelman avec une mise en garde à l'endroit de ceux qui voudraient procéder par ce truchement à du "chantage litigieux" ([1985] 1 RCS 2 à la p 37, 15 DLR (4e) 161). Sur la fin de non- 
parce que le droit est parfois moins impuissant qu'il n'y paraît. On aurait pu aussi se risquer à parler d'arme conventionnelle, pour tout le droit des contrats, ou à dire du droit qu'il est un canon, puisqu'il est canonique, c'est-à-dire normatif.

Sauf qu'il y a un hic, avec cette image de l'arme, une faille radicale. Le loup, même avec des gants de soie, garde la dent longue. Dès lors, l'image de l'arme est perverse en ce qu'elle sape le fondement même du droit en choquant de sa violence l'idée de civilisation et de concorde dont il est, sinon facteur, assurément porteur. On l'a dit ce matin², c'est sans armes que le droit tient la force au respect ${ }^{3}$.

D'accord. Mais si le droit n'est pas une arme, qu'est-il donc?

D'instinct, on oppose l'arme à l'outil. Là où l'arme divise, l'outil assemble. En admettant que l'arme assure la défense de l'homme, et je n'en suis pas certaine, c'est aussi une fonction de l'outil, qui en plus soutient et abrite. Avec la truelle et le marteau, on bâtit des préaux et des prétoires, des maisons et des musées. Les clés tiennent des voûtes! Les coins sont propices à la réflexion! Sans ciseau, l'ange, hélas, demeure prisonnier du marbre4. L'aiguille encore raccommode, peut-être la robe de saint Yves, peut-être des tissus sociaux. Et la règle? Elle apprend à connaître le monde, l'arpente, le sonde, le mesure. Règle de plomb de l'équité ${ }^{5}$, règles d'or de l'aspiration.

recevoir et l'abus de droit de bouclier, voir Combe v Combe, [1951] 2 KB 215, [1951] All ER 767 (CA).

2 Voir Imed Ben Cheikh Larbi, Conférence internationale des barreaux de tradition juridique commune, présentée au Palais des Congrès de Cotonou, 3 décembre 2015 [non publiée].

3 Voir François Fyot de la Marche, L'Éloge et les devoirs de la profession d'avocat, Paris, Nicolas Mazuel, 1713 à la p 28 (" [s]ans armes [cette profession] épouvante la force, sans effort elle arrête la violence, sans appareil elle réduit la puissance et le faste à la modestie et à la crainte »).

4 Voir cette citation, attribuée à Michel-Ange, en référence à son œuvre La Pietà : « J'ai vu un ange dans le marbre et j'ai seulement ciselé jusqu'à l'en libérer ".

5 Voir Aristote, Éthique à Nicomaque, 5e éd, traduit par Jules Tricot, Paris, J Vrin, 1983, aux pp 1137b-1138a :

De ce qui est, en effet, indéterminé la règle aussi est indéterminée, à la façon de la règle de plomb utilisée dans les constructions de Lesbos : de même que la règle épouse les contours de la pierre et n'est pas rigide, ainsi le décret est adapté aux faits.

On voit ainsi clairement ce qu'est l'équitable, que l'équitable est juste et qu'il est supérieur à une certaine sorte de juste. De là résulte nettement aussi la nature de l'homme équitable : celui qui a tendance à choisir et à accomplir les actions équitables et ne s'en tient pas rigoureusement à ses droits dans le sens du pire, mais qui a tendance à prendre moins que son dû, bien qu'il ait 
Certes, tout outil peut devenir une arme, la rose pique, le papier coupe, et quelques crimes déjà ont dénaturé l'anodin en mortifère ${ }^{6}$. Mais je refuse de voir Landru dans tous les fourneaux. Ma main tendue vers vous n'est pas une arme. À telle enseigne, dire du droit que c'est une arme, c'est faire de chaque bâton un épieu plutôt qu'un levier; c'est voir un billot dans chaque bûche plutôt que la chaleur d'un foyer — vous excuserez l'image nordique - c'est voir sainte Catherine dans toutes les roues, plutôt qu'un symbole de progrès, alors qu'à la roue, il faut mettre l'épaule.

Qui a vu dans l'éclat du silex l'éclat du génie et l'étincelle d'un premier feu a su aussi qu'il devrait y veiller. Mais celui-là a pu voir encore dans la flèche qu'il s'appliquait à tailler, la direction du progrès. Car l'outil, comme le droit, suppose un travail, et de longue haleine. Par contraste, l'arme est à saisir, vivement, il faut s'en emparer; toute guerre est à finir; la culture, elle, tend toujours aux lendemains.

Oui voilà, alors que la civilisation veut rester, les armes, elles, veulent être prises. Dire du droit que c'est une arme, c'est suggérer que l'on puisse se l'approprier. Or, je refuse un droit appropriable, c'est-à-dire un droit réduit à la préhension, à l'appréhension, à la raison du plus fort. Je veux un droit de compréhension, c'est-à-dire que l'on puisse prendre avec et porter en soi.

Un droit d'échanges, pas un droit bouclier. Le droit que je vois n'est pas levé, il élève. Il protège non comme une carapace isole, mais comme un mécène, un tuteur qui guide, montre, qui présente au cénacle. Ma main tendue. Votre main tendue, une poignée de main plutôt qu'un poing brandi, fermé.

Or, le droit est ouvert. Dire du droit que c'est une arme, c'est suggérer qu'il faille le garder hors de la portée des enfants. Je veux un droit auquel puissent venir aussi les petits enfants7, et par "petits " j'entends chacun

la loi de son côté, celui-là est un homme équitable, et cette disposition est l'équité, qui est une forme spéciale de la justice et non pas une disposition entièrement distincte [notes omises].

6 Timothy McVeigh avait utilisé de l'engrais agricole; adieu Cherbourg, le parapluie de Markov aussi est une arme.

7 Voir dans Nouveau Testament, Paris, Cerf, 1977, Évangile selon saint Marc, 10, 13-16 :

Des gens lui amenaient des enfants pour qu'il les touche, mais les disciples les rabrouèrent. En voyant cela, Jésus s’indigna et leur dit : « Laissez les enfants venir à moi, ne les empêchez pas, car le Royaume de Dieu est à ceux qui sont comme eux. En vérité je vous le déclare, qui n'accueille pas le Royaume de Dieu comme un enfant n'y entrera pas ». Et il les embrassait et les bénissait en leur imposant les mains.

Voir aussi ibid, Évangile selon saint Luc, 18, 15-17. 
de nous, unités fractionnaires du tout social ${ }^{8}$, chacun dans sa chacunité, chacun comme médiation de rêves et de défis ${ }^{9}$, expression délicate de fantasmes et d'exigences ${ }^{10}$. "Les hommes sont limités", disait Kourouma, "ils ne réussissent pas des œuvres infinies " ${ }^{11}$. Chacun, peut-être, mais ensemble, leur œuvre est sans limites. Fraternité, justice, travail ${ }^{12}$. Le droit est ainsi une stratégie concertée pour le possible ${ }^{13}$.

Et pour cela, l'image de l'outil, si elle reflète mieux l'impulsion de civilisation du droit, n'est pas complètement satisfaisante. L'image est plus juste que celle de l'arme à deux titres, d'abord parce qu'elle exprime la polyvalence des fonctions du droit - l'outil transformé en arme, c'est déjà plus que l'arme - ensuite parce que la conversion ${ }^{14}$ de l'outil en arme re-

8 Voir Jean-Jacques Rousseau, Émile ou de l'éducation, Paris, Éditions Sociales, 1958 à la p 90 :

L'homme naturel est tout pour lui; il est l'unité numérique, l'entier absolu, qui n'a de rapport qu'à lui-même ou à son semblable. L'homme civil n'est qu'une unité fractionnaire qui tient au dénominateur, et dont la valeur est dans son rapport avec l'entier, qui est le corps social. Les bonnes institutions sociales sont celles qui savent le mieux dénaturer l'homme [...] en sorte que chaque particulier ne se croie plus un, mais partie de l'unité, et ne soit plus sensible que dans le tout [notes omises].

9 Voir Jacques Vanderlinden, «Harmonisation et dissonance : langues et droit au Canada et en Europe ou de l'intérêt éventuel des synthèses " (2000) 3:1-2 RCLF 217 aux pp 266-67.

10 Voir Sergio Cotta, Ontologie du phénomène juridique, Paris, Dalloz, 2015 à la p 49 (« [1]e juge juste n'est donc pas un fantasme, mais une exigence au sens fort du mot»). Pour une définition, voir Centre national de ressources textuelles et lexicales, sub verbo " exigence», en ligne : <www.cnrtl.fr/definition/exigence> (« [c]e que l'on exige. [...] Ce que l'on veut impérativement. [...] P. ext. Ce que l'on attend impérativement de quelqu'un ").

11 Ahmadou Kourouma, Monnè, outrages et défis, Paris, Seuil, 1990 à la p 28.

12 Devise nationale du Bénin.

13 Voir Romain Gary, La promesse de l'aube, Paris, Gallimard, 1960 aux pp 95-96 :

Mais il est encore plus difficile de se résigner. Combien de fois me suis-je trouvé, depuis mes débuts dans la carrière d'artiste, la plume à la main, plié en deux, accroché au trapèze volant, les jambes en l'air, la tête en bas, lancé à travers l'espace, les dents serrées, tous les muscles tendus, la sueur au front, au bout de l'imagination et de la volonté, à la limite de moi-même, cependant qu'il faut encore conserver le souci du style, donner une impression d'aisance, de facilité, paraître détaché, au moment de la plus intense concentration, léger au moment de la plus violente crispation, sourire agréablement, retarder la détente et la chute inévitable, prolonger le vol, pour que le mot "fin" ne vienne pas prématurément comme un manque de souffle, d'audace et de talent, et lorsque vous voilà enfin de retour au sol, avec tous vos membres miraculeusement intacts, le trapèze vous est renvoyé, la page redevient blanche, et vous êtes prié de recommencer.

14 Voir Jules Vallès, "Paris vendu », éditorial, Le Cri du Peuple (22 février 1871) : "La [guerre] [s]ociale arrive, entendez-vous! elle arrive à pas de géant, apportant, non la 
flète le rôle de la volonté de l'homme, suppose une intention, un choix, et donc, traduit son libre arbitre. Cependant, l'image se heurte aux mêmes limites matérielles. L'outil est une extension de l'homme, peut-être, mais non son dépassement.

Or, le droit est un trait entre le matériel et l'aspiration ${ }^{15}$, entre ce qui peut et ce qui doit, entre l'ange et le marchand, entre les valeurs-idéaux et les valeurs-prix, entre la tête qui pense et la panse qui gronde ou, plus subtil, entre le cœur qui bat et le bras qui besogne.

Le droit est un corps complexe, écheveau de volontés, d'intérêts, de rapports et de règles. Le droit est un réseau. Le droit est un transport. Le droit est un acte de sortir-de-soi.

Celui qui met en joue est sûr de son droit. Il s'est soustrait au doute en s'emparant d'un fusil. Devant un droit bigarré et pluriel, cette certitude détonne ${ }^{16}$.

Si l'homme se savait infaillible, il ne communiquerait pas. Mettons Descartes sur table, le fondement du discours, c'est le doute. Apostrophes et répliques, questions et réponses sont les balises de la circulation des idées, de la route qui mène à l'intelligence, comprise ici au sens de bonne entente. Le droit, c'est l'accord indispensable entre des propositions polyvoques, qui les stabilise dans l'harmonie. Errantes qu'elles étaient, elles se fixent et deviennent, in-errantes, inhérentes, c'est-à-dire, par définition, essentielles et nécessaires ${ }^{17}$.

mort, mais le salut. Elle enjambe par-dessus les ruines, et elle crie! "Malheur aux traîtres! malheur aux vainqueurs!". Vous espérez l'assassiner. Essayez! Debout entre l'arme et l'outil, prêt au travail ou à la lutte, le Peuple attend".

15 Voir Robert M Cover, "Bringing the Messiah Through the Law: A Case Study" dans J Roland Pennock et John W Chapman, dir, Religion, Morality, and the Law, New York, New York University Press, 1988, 201 à la p 201.

16 Voir Napoléon, Correspondance de Napoléon Ier, t 31, Paris, Imprimerie impériale, 1869 à la p 378 (« [l]e tambour imite le bruit du canon; c'est le meilleur de tous les instruments; il ne détonne jamais »).

17 Voir Centre National de Ressources Textuelles et Lexicales, sub verbo "inhérent", en ligne : <www.cnrtl.fr/definition/inhérent> (« [q]ui, de par sa nature, est lié d'une manière inséparable et nécessaire à une personne ou à une chose »); Cotta, supra note 10 à la p 33 :

L'argumentation a précisément le but de faire naître entre nous cet accord indispensable, en établissant des propositions inerrantes, un dire inerrant et donc infaillible (dans le sens de: sans faute, sans erreur). Mais d'autre part, nous recourons à l'argumentation précisément parce que nous doutons, c'est-à-dire parce que nous savons (avec certitude, donc infailliblement!) que nous sommes faillibles. Si les hommes étaient infaillibles, ils n'argumenteraient point, ils ne communiqueraient même pas dans le mode du discours : ils s'identifieraient en et avec la vérité. 
Et la voilà l'image.

Le droit est un espace. Habité, meublé, travaillé par l'homme. Euvre et trame de nos débats, tantôt enflammés, tantôt sereins, c'est une place publique, un carrefour, un espace libre. Espace en quatre temps.

Le droit est d'abord un espace politique, un acte de volonté législative, de manière évidente dans les traditions de droit écrit, mais aussi, je l'ai dit, un geste discursif, à la fois expression d'une communauté ${ }^{18}$ et tremplin d'une multitude d'exposés. Comme le disait Portalis, "les lois, une fois rédigées, demeurent telles qu'elles ont été écrites; les hommes, au contraire, ne se reposent jamais; ils agissent toujours ${ }^{19}$.

Le droit, ensuite, est un espace commun. Le Code en est l'archétype. Le mien, le Code civil du Québec, le dit d'ailleurs lui-même et d'entrée de jeu, il établit, non pas le droit privé mais bien le droit commun ${ }^{20}$. En légiférant des matières pratiques et ordonnées - personnes, familles, successions, biens, obligations - il inscrit le respect de tous et la primauté de chacun, établit l'égalité devant la loi et dans les rapports humains et consacre l'autonomie et la liberté21. Ce n'est pas peu, c'est même beaucoup.

Le droit, encore, est un espace infini, infini comme celui de la bibliothèque de Babel qui, entre ses quatre murs, contiendrait un nombre infini de livres, qui pourraient avoir un nombre infini de pages ${ }^{22}$, qui porteraient un nombre infini d'histoires. Infini et incommensurable, tantôt intime, qui parle de consentement et des liens du sang, et, tantôt surdimensionné,

18 Voir Jean-Guy Belley, «Présentation » dans Serge Lortie, Nicholas Kasirer et Jean-Guy Belley, dir, Du Code civil du Québec : contribution à l'histoire immédiate d'une recodification réussie, Montréal, Thémis, 2005, 1 aux pp 7, 18; Jean Pineau, «La réforme d'un Code civil » dans Lortie, Kasirer et Belley, supra note 18, 233 aux pp 267-71.

19 Jean-Étienne-Marie Portalis, « Discours préliminaire sur le projet de Code Civil présenté le $1^{\text {er }}$ pluviose en IX par la Commission nommée par le gouvernement consulaire" dans Jean-Étienne-Marie Portalis, Discours et rapports sur le Code Civil : précédés de $l$ 'Essai sur l'utilité de la codification de Frédéric Portalis, Caen, Presses universitaires de Caen, 2010 à la p 66.

20 Voir Alain-François Bisson, "La Disposition préliminaire du Code civil du Québec» (1999) 44:3 RD McGill 539 aux pp 551-53.

21 Voir James Oliver Murdock, "Le Code civil français vu par un Américain » (1954) 6:4 RIDC 678 à la p 678 (" [e]n même temps qu'il légifère sur des questions prosaïques de droit privé - les personnes, les biens et les différentes manières dont on acquiert la propriété - il établit l'égalité devant la loi et la liberté individuelle. Il protège les entreprises privées et la propriété. C'est là un record inégalé de concentration").

22 Voir Jorge Luis Borges, "La bibliothèque de Babel» dans Jorge Luis Borges, Euvres complètes, traduit par Paul Bénichou et al, Gallimard, 2010, vol 1, 491 [Borges, Euvres complètes]. Voir aussi Jorge Luis Borges, "Le livre de sable", vol 2 dans Borges, Euvres complètes, supra note 22, 550 . 
herculéen ${ }^{23}$, qui organise la république et qui épouse la primauté du droit. La page du livre de loi en a peut-être long et large, mais le droit en a aussi profond. C'est cela, l'esprit des lois.

Enfin, le droit est un espace carré, carrant. Il structure, il ossature, il armature. Un espace carré, c'est-à-dire aménagé, par des conceptions, sociales, morales, philosophiques, culturelles, économiques même ${ }^{24}$. C'est un espace modelé, stylé, élégant ${ }^{25}$, en l'occurrence, ici, il a le style de notre tradition juridique commune et l'élégance de notre langue en partage. Le droit est encore une aire de jeu, un espace de ludicité, solide et solidaire, syncrétique. Le droit est un espace de partage, une plate-forme essentielle, une passerelle propice. Le droit est un trait, disais-je, le droit est un pont $^{26}$. Le droit est un arbre vivant ${ }^{27}$. Le droit est un jardin public ${ }^{28}$, peutêtre, où tout peut fleurir et où chacun peut oser. En somme, $\delta$ c'est un jardin extraordinaire. J Il suffit pour ça d'un peu d'imagination ${ }^{29}$.

Et Giraudoux nous aura rappelé à nous, juristes, combien le droit, en est « la plus puissante des écoles $»^{30}$. L'imagination, c'est le possible, c'est

23 Voir Ronald Dworkin, Law's Empire, Cambridge (Mass), Harvard University Press, 1986.

24 Voir Pineau, supra note 18 à la p 267 et $\mathrm{s}$.

25 Voir généralement Nicholas Kasirer, dir, Le droit civil, avant tout un style?, Montréal, Thémis, 2003 [Kasirer, Le droit civil]. Voir notamment Marie José Longtin, « Le style civiliste et la loi » dans Kasirer, Le droit civil, supra note 25, 185. Voir aussi, bien sûr, la Lettre de Marie-Henry Beyle dit Stendhal à Honoré de Balzac (30 octobre 1840) dans A Paupe et P-A Cheramy, dir, Correspondance de Stendhal (1800-1842), t 3, Paris, Charles Bosse, 1908, 257 à la p 259 : « En composant la Chartreuse, pour prendre le ton, je lisais chaque matin deux ou trois pages du Code civil, afin d'être toujours naturel; je ne veux pas, par des moyens factices, fasciner l'âme du lecteur». Mais bon, Stendhal était aussi du genre à s'évanouir devant la beauté porphyrienne des cathédrales.

26 Voir Renaud Payre et Gilles Pollet, Socio-histoire de l'action publique, Paris, La Découverte, 2013, ch 4-5.

27 Voir Edwards v Attorney-General for Canada (1929), [1930] AC 124 à la p 136, [1930] 1 DLR 98 (PC) :

The British North America Act planted in Canada a living tree capable of growth and expansion within its natural limits. The object of the Act was to grant a Constitution to Canada. "Like all written constitutions it has been subject to development through usage and convention" [références omises].

28 Le droit est un jardin de curé, c'est-à-dire « bien entretenu, cultivé de façon rationnelle et comprenant une grande variété de plantes" (Centre national de ressources textuelles et lexicales, sub verbo " jardin ", en ligne : <www.cnrtl.fr/definition/jardin>).

29 Voir Charles Trenet, Le jardin extraordinaire (1957).

30 Jean Giraudoux, La Guerre de Troie n'aura pas lieu, Paris, Grasset, 1935, acte II, scène $\mathrm{V}$ à la $\mathrm{p} 121$ (" [m]on cher Busiris, nous savons tous ici que le droit est la plus puissante des écoles de l'imagination. Jamais poète n'a interprété la nature aussi librement qu'un juriste la réalité »). 
œuvrer de hardiesse; c'est que le droit est intrépide ${ }^{31}$. Le droit est-il une arme? Non, le droit est, désarmant.

31 Voir Centre national de ressources textuelles et lexicales, sub verbo "intrépide", en ligne: <www.cnrtl.fr/definition/intrepide> («[q]ui ne tremble pas, qui demeure ferme devant le danger. [...] Qui manifeste, qui dénote ou qui implique une fermeté ou une détermination résolue. [...] $P$. ext. [...] Qui est ferme dans sa conduite (dans l'action, le comportement que dénote le nom), qui n'est pas découragé par les difficultés, les obstacles; que rien ne rebute "). 